\title{
Impact of advance directives on the variability between intensivists in the decisions to forgo life-sustaining treatment
}

Margot Smirdec ${ }^{1}$, Mercé Jourdain ${ }^{2}$, Virginie Guastella ${ }^{3}$, Céline Lambert ${ }^{4}$, Jean-Christophe Richard ${ }^{5}$, Laurent Argaud ${ }^{6}$, Samir Jaber ${ }^{7}$, Kada Klouche $^{8}$, Anne Medard ${ }^{9}$, Jean Reignier ${ }^{10}$, Jean-Philippe Rigaud ${ }^{11}$, Jean-Marc Doise ${ }^{12}$, Russell Chabanne ${ }^{13}$, Bertrand Souweine ${ }^{14}$, Jeremy Bourenne ${ }^{15}$, Julie Delmas ${ }^{16}$, Pierre-Marie Bertrand ${ }^{17}$, Philippe Verdier ${ }^{18}$, Jean-Pierre Quenot ${ }^{19}$, Cecile Aubron ${ }^{20}$, Nathanael Eisenmann ${ }^{21}$, Pierre Asfar ${ }^{22}$, Alexandre Fratani ${ }^{23}$, Jean Dellamonica ${ }^{24}$, Nicolas Terzi ${ }^{25}$, Jean-Michel Constantin ${ }^{26}$, Axelle Van Lander ${ }^{27,28}$, Renaud Guerin ${ }^{29}$, Bruno Pereira ${ }^{4}$ and Alexandre Lautrette $21,30,31^{*}$ (B)

\begin{abstract}
Background: There is wide variability between intensivists in the decisions to forgo life-sustaining treatment (DFLST). Advance directives (ADs) allow patients to communicate their end-of-life wishes to physicians. We assessed whether ADs reduced variability in DFLSTs between intensivists.

Methods: We conducted a multicenter, prospective, simulation study. Eight patients expressed their wishes in ADs after being informed about DFLSTs by an intensivist-investigator. The participating intensivists answered ten questions about the DFLSTs of each patient in two scenarios, referring to patients' characteristics without ADs (round 1) and then with (round 2). DFLST score ranged from 0 (no-DFLST) to 10 (DFLST for all questions). The main outcome was variability in DFLSTs between intensivists, expressed as relative standard deviation (RSD).
\end{abstract}

Results: A total of 19,680 decisions made by 123 intensivists from 27 ICUs were analyzed. The DFLST score was higher with ADs than without $(6.0295 \% \mathrm{Cl}[5.85 ; 6.19]$ vs $4.9295 \% \mathrm{Cl}[4.75 ; 5.10], p<0.001)$. High inter-intensivist variability did not change with ADs (RSD: 0.56 (round 1) vs 0.46 (round 2), $p=0.84$ ). Inter-intensivist agreement on DFLSTs was weak with ADs (intra-class correlation coefficient: 0.28). No factor associated with DFLSTs was identified. A qualitative analysis of ADs showed focus on end-of-life wills, unwanted things and fear of pain.

Conclusions: ADs increased the DFLST rate but did not reduce variability between the intensivists. In the decisionmaking process using ADs, the intensivist's decision took priority. Further research is needed to improve the matching of the physicians' decision with the patient's wishes.

Trial registration ClinicalTrials.gov Identifier: NCT03013530. Registered 6 January 2017; https://clinicaltrials.gov/ct2/ show/NCT03013530.

\footnotetext{
*Correspondence: alautrette@chu-clermontferrand.fr

${ }^{21}$ Intensive Care Unit, Centre Jean Perrin, 54 Rue Montalembert, BP69, 63003 Clermont-Ferrand, Cedex 1, France

Full list of author information is available at the end of the article
}

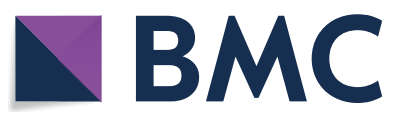

(c) The Author(s) 2020. Open Access This article is licensed under a Creative Commons Attribution 4.0 International License, which permits use, sharing, adaptation, distribution and reproduction in any medium or format, as long as you give appropriate credit to the original author(s) and the source, provide a link to the Creative Commons licence, and indicate if changes were made. The images or other third party material in this article are included in the article's Creative Commons licence, unless indicated otherwise in a credit line to the material. If material is not included in the article's Creative Commons licence and your intended use is not permitted by statutory regulation or exceeds the permitted use, you will need to obtain permission directly from the copyright holder. To view a copy of this licence, visit http://creativecommons.org/licenses/by/4.0/. The Creative Commons Public Domain Dedication waiver (http://creativeco mmons.org/publicdomain/zero/1.0/) applies to the data made available in this article, unless otherwise stated in a credit line to the data. 
Keywords: Advance directives, Decisions to forgo life-sustaining treatment, ICU

\section{Background}

A decision to forgo life-sustaining treatment (DFLST) is made by $3-30 \%$ of intensive care unit (ICU) patients and is recorded in $90 \%$ of decedent patients [1]. The DFLST includes decisions about no-escalation or withholding or withdrawal of treatment that lead to differences in mortality [2]. These decisions are made by the patient, the physician or close relatives, or result from a shared decision-making process. However, there are numerous limitations to this process. A DFLST made by the family can be influenced by their preferences [3] or by the psychological symptoms associated with ICU admission such as anxiety/depression and post-traumatic stress disorder, which prevent the patient's wishes being clearly reported [4]. In addition, misperceptions about the patient's prognosis by the surrogate can lead to differing expectations by physicians and family and delay decision-making [5, 6]. When making a DFLST, physicians are greatly influenced by their personal characteristics including religion and culture [7,8], which results in considerable variability in their decisions $[7,9]$. This variability is constant within the same specialty or structure [10-14]. Patients want physicians to follow their wishes [15], but most ICU patients are not able to properly communicate these wishes because they lack decision-making capacity. Advance directives (ADs) give incapacitated patients the opportunity to indicate what treatment they wish to have [16]. There is a worldwide consensus that physicians should respect the patient's ADs [17]. In a given setting, when the respect of the patient's wishes has priority over the personal opinion of the physician, ADs could lead to a decrease in variability in DFLSTs among physicians. However, there is no discussion between the patient and the physician to explain the wishes expressed in ADs. As a result, the physician may interpret the patient's wishes differently from what was intended. Our study assessed whether ADs, drawn up by patient after receiving information about DFLSTs and viewing a related video with an intensivist-investigator, would reduce variability in DFLST between intensivists compared to decision-making without ADs. The other aim of the study was to identify the factors associated with DFLSTs or with change in DFLSTs when ADs were available.

\section{Methods}

We conducted a multicenter, prospective, simulation study, in France from September 2017 to March 2018. The study was approved by the local French ethics committee (Comité de Protection des Personnes Sud-Est VI de Clermont-Ferrand (IRB00008526; No. 2016/CE87). A consent form was collected from all participants (patients and intensivists) after they had been informed orally and received a written information form. The study was registered on the ClinicalTrials.gov website under number: NCT03013530 in January 2017 and complied with the guidelines of Strengthening the Reporting of Observational Studies in Epidemiology (STROBE) [18].

\section{Patients and advance directives}

The patients participating in the study were selected from the cohort of consecutive outpatients seen during January-March 2017 for follow-up of chronic disease in the cardiology, pulmonology or nephrology departments of the University Hospital of Clermont-Ferrand. The patient selection criteria were severe stage of chronic cardiac or kidney or respiratory failure associated with comorbid conditions, and life expectancy of less than 5 years according to McCabe score [19], but without acute episode or cognitive impairment on the basis of criteria described by Appelbaum [20]. Of the 23 patients selected, 1 died before the meeting with the intensivistinvestigator, 1 was transferred to the palliative care unit, 13 declined and 8 accepted to participate in the study. The characteristics of the 8 patients are shown in Additional file 1. One intensivist-investigator (MS) met each of the eight patients at their home or at a hospital office to provide personalized, clear and full information about DFLSTs and ADs using a video and to suggest that they draw up ADs. The intensivist-investigator had 5 years of ICU practice experience and a master's diploma in ethics. She was trained in communication skills and in faceto-face conversation about ADs. The video, which lasted 10 min, explained ICU life-sustaining treatment, DFLSTs, and the objectives of ADs as laid out in the guidelines of the French Health Authority. During the meeting, the AD forms of the French Health Authority were given to the patient, who had the opportunity to ask questions about DFLSTs, ADs or end-of-life. The patients were then asked questions to check they had understood the information given. The $\mathrm{AD}$ forms comprise 11 pages in which patients can designate a surrogate, express their wishes about lifesustaining treatments in the free-text boxes and 15 pages of guidelines about the writing of wishes, and the use of ADs by physicians in accordance with the French law of 2016 [21], which requires physicians to comply with the patients' wishes except if the ADs are obviously inappropriate [22]. At the end of the meeting, the patients were invited to draw up their ADs or to take time to discuss 
with relatives. One patient made a second meeting with the intensivist-investigator to ask further questions. The median time of the meetings was $85.6[60 ; 120] \mathrm{min}$. The ADs were returned within 1 month of the meeting.

\section{Study procedure}

Two clinical scenarios were created by a multidisciplinary team made up of two physicians in palliative care and three intensivists who did not participate in the study. Scenario 1 was followed by six questions and Scenario 2 by four, and both investigated the use of life-sustaining treatment (ICU admission, intubation, renal replacement therapy, vasoactive drugs, tracheotomy) for community-acquired pneumonia with septic shock and for septic shock after gastrointestinal surgery, respectively (see Additional file 2). The possible replies were "yes" or "no" for six questions and "yes" or "partially" or "no" for four questions. The replies were rated $1,0.5$ or 0 if they corresponded to a DFLST, a partial DFLST or no DFLST, respectively. The sum of these ten replies made up a DFLST score ranging from 0 if there was no DFLST to 10 if there was DFLST for all questions. The two scenarios were submitted online to intensivists from 27 French ICUs in 14 university hospitals and in 9 general hospitals. Each intensivist independently and anonymously completed the questionnaires of the two scenarios for each of eight patients with only the patient's characteristics available (round 1) and then with the same characteristics and the patient's ADs available (round 2). Round 2 was submitted to intensivists 2 weeks after completion of round 1 (see Additional file 3).

\section{Statistical analysis}

The primary endpoint was variability in DFLSTs between intensivists when ADs were available (round 2) and when they were not (round 1) expressed as the relative standard deviation (RSD; range $0-1$; a high rate indicates high inter-intensivist variability). The second endpoint was the identification of factors associated with the DFLSTs or with changes in DFLSTs when ADs were provided. The patient characteristics given to intensivists for the two rounds were age, sex, housing, relatives, medical history, comorbid conditions defined as all diseases or trauma current or previous with the stage or complications reported by the patient or retrieved from medical chart, treatments and dependence in activities of daily living [23]. The recorded characteristics of the intensivists were age, sex, status of intensivist, length of practical experience, speciality of anesthesiology and critical care, religion, interest in ethics, traumatic experience of an end-of-life situation, type of hospital, type of ICU, number of beds and number of intensivists in the ICU,
DFLST protocol available in ICU and number of DFLSTs per week.

The number of patients participating in the study was fixed according to the feasibility of answering 20 questions per patient for each round and the case-mix. The sample size of intensivists was fixed to assess how ADs affected the change in variability between intensivists in the DFLSTs. We calculated that at least 120 intensivists evaluating eight patients were necessary to show a $20 \%$ relative difference of variability (sample size for a twosample standard deviations $\mathrm{F}$ test), for a two-sided type I error at 5\%, a statistical power of $90 \%$ and an intra-class correlation coefficient at 0.5, i.e., 960 DFLST scores per round.

All statistical analyses were performed with Stata software (version 15, StataCorp, College Station USA, TX). Continuous data were expressed, according to statistical distribution, as mean and standard-deviation or median and interquartile range. The assumption of normality was studied using the Shapiro-Wilk test. Changes in DFLSTs were compared between rounds 1 and 2 with randomeffects models taking into account between and within intensivist variability (intensivists as random-effect). Pitman's test was used to compare RSD. The agreement on DFLSTs between intensivists (inter-agreement) and the within intensivists agreement (intra-agreement) on DFLSTs between the two rounds for each patient were studied using intra-class correlation coefficient (ICC) estimated by the mixed models mentioned above. ICC was interpreted according to standard recommendations: $<0.2$ (negligible agreement), 0.20-0.39 (weak agreement), 0.40-0.59 (moderate agreement), 0.60-0.79 (good agreement) and $\geq 0.8$ (excellent agreement).

To determine the characteristics of the ICU physicians associated with DFLSTs in round 1 or with the change in DFLSTs when ADs were provided, random-effects models (i.e., linear mixed models) were carried out with the following parameters as fixed covariates: age, sex, status of intensivist, type of hospital, type of ICU, length of practice and religion. The session effect associated with the two measurement times was studied in the same way. The results are expressed in terms of standardized mean differences and 95\% confidence intervals. All analyses were performed with a two-sided type I error of $5 \%$. No missing data were observed.

To evaluate the representativeness of the participating intensivists, a sensitivity analysis was performed to compare their characteristics with those of the non-participating intensivists. Non-participating intensivists were intensivists contacted but who did not reply to all the questions of the two rounds.

A qualitative analysis of ADs was made by a multidisciplinary team composed of two intensivists, one 
psychologist, one physician in palliative care and two biostatisticians using Alceste software (IMAGE, CNRS, France).

\section{Results}

Of the 170 intensivists contacted, 123 from 27 ICUs completed the two rounds and made up the study population. Their demographic characteristics are given in Table 1 . There was no difference between the study population and non-participating intensivists. A total of 19,680 intensivists' decisions from the two rounds were analyzed.

\section{Impact of ADs on the DFLSTs and on the variability in DFLSTs}

The proportions of DFLSTs for rounds 1 and 2 are shown in Fig. 1a, b.
The DFLST score was higher with ADs (round 2) than without ADs (round 1) $(6.0295 \%$ CI [5.85; 6.19] vs 4.92 $95 \%$ CI $[4.75 ; 5.10], p<0.001$ ) (Table 2 ). There was no significant change in inter-intensivists variability when ADs were provided (RSD: 0.56 (round 1) vs 0.46 (round 2), $p=0.84$ ) (Table 2). In three patients, the RSD decreased but remained high (Table 2). Inter-intensivists agreement on DFLSTs was moderate $(\mathrm{ICC}=0.42)$ without ADs (round 1) and weak (ICC $=0.28$ ) with ADs (round 2). Intra-intensivists agreements on DFLSTs between the two rounds ranged from weak $(\mathrm{ICC}=0.22)$ to moderate $(\mathrm{ICC}=0.56)$ (Table 2).

\section{Identification of factors associated with the DFLSTs} and with the change in DFLSTs when ADs were available In the univariate analyses of the overall data of the eight patients, no factor associated with the DFLST score in

Table 1 Characteristics of intensivists

\begin{tabular}{|c|c|c|c|c|}
\hline Variable & $\begin{array}{l}\text { Intensivists contacted } \\
n=170\end{array}$ & $\begin{array}{l}\text { Participating } \\
\text { intensivists } \\
n=123\end{array}$ & $\begin{array}{l}\text { Non-participating } \\
\text { intensivists } \\
n=47\end{array}$ & $p$ value \\
\hline Male gender, n (\%) & $113(63.8)$ & $84(68.3)$ & $26(55.3)$ & 0.11 \\
\hline Age (years), mean \pm sd & $39.9 \pm 8.5$ & $40.0 \pm 8.5$ & $39.8 \pm 8.6$ & 0.88 \\
\hline \multicolumn{5}{|l|}{ Status of intensivist, n (\%) } \\
\hline Assistant & $37(21.8)$ & $24(19.5)$ & $13(27.7)$ & \\
\hline Senior & $105(61.8)$ & $78(63.4)$ & $27(57.5)$ & 0.51 \\
\hline Professor & $28(16.4)$ & $21(17.1)$ & $7(14.8)$ & \\
\hline Length of overall professional experience (years), median [IQR] & $6[3 ; 15]$ & $7[3 ; 15]$ & $6[3 ; 15]$ & 0.61 \\
\hline$<5$ years, $\mathrm{n}(\%)$ & $60(35.3)$ & $43(35.0)$ & $17(36.2)$ & \\
\hline $5-15$ years, $n(\%)$ & $73(42.9)$ & $50(40.6)$ & $23(48.9)$ & 0.37 \\
\hline$>15$ years, $n(\%)$ & $37(21.8)$ & $30(24.4)$ & $7(14.9)$ & \\
\hline Specialty of anesthesiology and critical care & $101(59.4)$ & $73(59.3)$ & $28(59.6)$ & 0.98 \\
\hline \multicolumn{5}{|l|}{ Religion, n (\%) } \\
\hline Catholic & $66(38.8)$ & $47(38.2)$ & $19(40.4)$ & \\
\hline Protestant & $5(2.9)$ & $4(3.3)$ & $1(2.1)$ & \\
\hline Islam & $5(2.9)$ & $3(2.4)$ & $2(4.3)$ & 0.74 \\
\hline Other & $10(5.9)$ & $9(7.3)$ & $1(2.1)$ & \\
\hline None & $84(49.5)$ & $60(48.8)$ & $24(51.1)$ & \\
\hline Intensivists with an interest in ethics, n (\%) & $127(74.7)$ & $90(73.2)$ & $37(78.7)$ & 0.56 \\
\hline Intensivists with a traumatic experience of an EOL situation, n (\%) & $79(46.5)$ & $57(46.3)$ & $22(46.8)$ & 0.96 \\
\hline ICU in university hospital, n (\%) & $128(75.3)$ & $94(76.4)$ & $34(72.3)$ & 0.58 \\
\hline \multicolumn{5}{|l|}{ Type of ICU, n (\%) } \\
\hline Medical & $72(43.1)$ & $52(43.0)$ & $20(43.5)$ & \\
\hline Surgery & $20(12.0)$ & $14(11.6)$ & $6(13.0)$ & 0.96 \\
\hline Mixed & $75(44.9)$ & $55(44.4)$ & $20(43.5)$ & \\
\hline Number of beds in ICU, median [IQR] & $15[10 ; 18]$ & $15[10 ; 18]$ & $13[10 ; 18]$ & 0.19 \\
\hline Number of intensivists in ICU, median [IQR] & $7[5 ; 10]$ & $7[5 ; 10]$ & $7[5 ; 8]$ & 0.09 \\
\hline DFLST Protocol available in ICU, n (\%) & $112(65.9)$ & $77(62.6)$ & $35(74.5)$ & 0.14 \\
\hline \multicolumn{5}{|l|}{ Number of DFLST performed in ICU, n (\%) } \\
\hline$<1 /$ week & $75 / 158(47.5)$ & $53 / 119(44.5)$ & 22/39 (56.4) & 0.20 \\
\hline
\end{tabular}

$D F L S T$ decision to forgo life-sustaining treatment, EOL end-of life, ICU intensive care unit, IQR interquartile range 


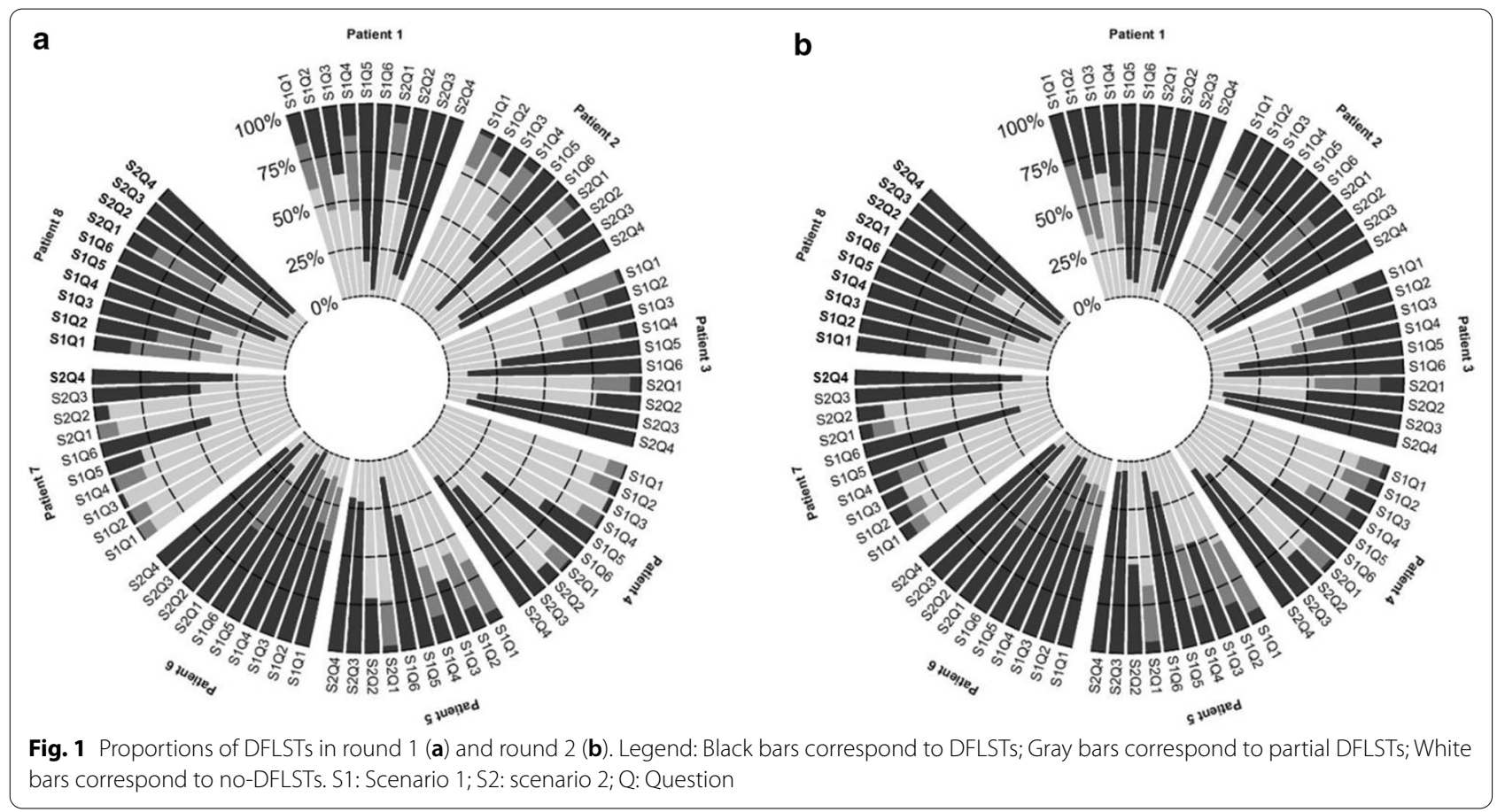

Table 2 Variability in the DFLST scores (RSD) and the within intensivist agreements (ICC)

\begin{tabular}{|c|c|c|c|c|c|c|c|}
\hline & \multicolumn{6}{|l|}{ DFLST score } & \multirow{3}{*}{$\begin{array}{l}\text { ICC } \\
\text { for intensivists }\end{array}$} \\
\hline & \multicolumn{3}{|l|}{ Median $[95 \% \mathrm{Cl}]$} & \multicolumn{3}{|l|}{ RSD } & \\
\hline & Round 1 & Round 2 & $p$ value & Round 1 & Round 2 & $p$ value & \\
\hline Patient \#1 & $5.63[5.20 ; 6.06]$ & $7.04[6.62 ; 7.47]$ & $<0.001$ & 0.43 & 0.34 & 0.82 & 0.48 \\
\hline Patient \#2 & $4.18[3.84 ; 4.52]$ & $6.31[5.86 ; 6.75]$ & $<0.001$ & 0.46 & 0.4 & 0.003 & 0.22 \\
\hline Patient \#3 & $4.69[4.29 ; 5.08]$ & $5.97[5.53 ; 6.42]$ & $<0.001$ & 0.47 & 0.42 & 0.18 & 0.47 \\
\hline Patient \#4 & $2.86[2.54 ; 3.18]$ & $3.84[3.45 ; 4.22]$ & $<0.001$ & 0.61 & 0.56 & 0.035 & 0.25 \\
\hline Patient \#5 & $4.70[4.25 ; 5.14]$ & $5.81[5.43 ; 6.19]$ & $<0.001$ & 0.53 & 0.36 & 0.08 & 0.38 \\
\hline Patient \#6 & $8.37[8.02 ; 8.71]$ & $8.16[7.76 ; 8.55]$ & 0.237 & 0.23 & 0.27 & 0.13 & 0.56 \\
\hline Patient \#7 & $2.42[2.12 ; 2.73]$ & $3.72[3.30 ; 4.14]$ & $<0.001$ & 0.71 & 0.63 & $<0.001$ & 0.24 \\
\hline Patient \#8 & $6.54[6.14 ; 6.95]$ & $7.32[6.88 ; 7.76]$ & 0.001 & 0.35 & 0.34 & 0.44 & 0.37 \\
\hline All patients & $4.92[4.75 ; 5.10]$ & $6.02[5.85 ; 6.19]$ & $<0.001$ & 0.56 & 0.46 & 0.84 & 0.56 \\
\hline
\end{tabular}

Cl confidence interval, DFLST decision to forgo life-sustaining treatment, RSD relative standard deviation, ICC: intra-class correlation coefficient

round 1 or with the change in the DFLST score when ADs were available was identified (Table 3 ).

\section{Qualitative analysis of ADs}

A qualitative analysis of ADs was performed on the verbatim texts amounting to 4091 words. Three themes, accounting for $59 \%$ of the words, emerged from the ADs: (1) wills of end-of-life and relatives, (2) unwanted things including therapeutic obstinacy and (3) fear of pain and loss of autonomy (Fig. 2). The most frequently occurring words were "life," "I" and "my." The word "death" was never stated. The patients expressed themselves in articulate well-structured sentences.

\section{Discussion}

Our results show that when provided with ADs, intensivists made more DFLSTs but this did not alter the great variability between them in their decisions. No factor associated with this variability was identified, suggesting multiple causes that were non-specific to the characteristics of the intensivists. In addition, the qualitative analysis of ADs highlighted the concerns expressed by the patient 
Table 3 Univariate analysis for the identification of factors associated with the DFLSTs and with the change in DFLSTs when advance directives were available

\begin{tabular}{|c|c|c|c|c|}
\hline Variable & $\begin{array}{l}\text { DFLST score in round } 1 \\
\text { Mean } \pm \text { SD } \\
\text { Correlation coefficient }\end{array}$ & $p$ value & $\begin{array}{l}\text { Change in DFLST score } \\
\text { between round } 1 \text { and round } 2 \\
\text { Mean } \pm \text { SD } \\
\text { Correlation coefficient }\end{array}$ & $p$ value \\
\hline \multicolumn{5}{|l|}{ Gender } \\
\hline Female & $5.06 \pm 2.72$ & 0.42 & $1.09 \pm 2.48$ & 0.96 \\
\hline Male & $4.86 \pm 2.80$ & & $1.10 \pm 2.43$ & \\
\hline Age (years) & 0.035 & 0.46 & 0.027 & 0.57 \\
\hline \multicolumn{5}{|l|}{ Status of intensivist } \\
\hline Assistant & $4.91 \pm 2.91$ & 0.74 & $0.98 \pm 2.48$ & 0.79 \\
\hline Senior & $4.98 \pm 2.74$ & & $1.15 \pm 2.42$ & \\
\hline Professor & $4.74 \pm 2.73$ & & $1.06 \pm 2.53$ & \\
\hline \multicolumn{5}{|c|}{ Length of overall professional experience } \\
\hline$<5$ years & $4.86 \pm 2.81$ & 0.23 & $1.18 \pm 2.45$ & 0.42 \\
\hline $5-15$ years & $4.77 \pm 2.72$ & & $0.94 \pm 2.33$ & \\
\hline$>15$ years & $5.27 \pm 2.79$ & & $1.23 \pm 2.63$ & \\
\hline \multicolumn{5}{|c|}{ Specialty of anesthesiology and critical care } \\
\hline Yes & $4.98 \pm 2.76$ & 0.62 & $1.09 \pm 2.46$ & 0.75 \\
\hline No & $4.86 \pm 2.81$ & & $1.15 \pm 2.42$ & \\
\hline \multicolumn{5}{|l|}{ Religion } \\
\hline Catholic & $4.94 \pm 2.74$ & 0.74 & $1.18 \pm 2.37$ & 0.78 \\
\hline Protestant & $4.75 \pm 2.61$ & & $0.78 \pm 1.90$ & \\
\hline Islam & $4.06 \pm 2.58$ & & $0.73 \pm 2.97$ & \\
\hline Other & $4.68 \pm 3.06$ & & $0.79 \pm 2.75$ & \\
\hline None & $5.00 \pm 2.77$ & & $1.12 \pm 2.46$ & \\
\hline \multicolumn{5}{|c|}{ Intensivists with an interest in ethics } \\
\hline Yes & $5.01 \pm 2.80$ & 0.22 & $1.14 \pm 2.49$ & 0.52 \\
\hline No & $4.69 \pm 2.70$ & & $0.99 \pm 2.34$ & \\
\hline \multicolumn{5}{|c|}{ Intensivists with a traumatic experience of an EOL situation } \\
\hline Yes & $4.79 \pm 2.73$ & 0.27 & $1.27 \pm 2.44$ & 0.09 \\
\hline No & $5.04 \pm 2.80$ & & $0.95 \pm 2.45$ & \\
\hline \multicolumn{5}{|l|}{ Hospital type } \\
\hline General & $4.74 \pm 2.93$ & 0.37 & $1.33 \pm 2.43$ & 0.18 \\
\hline University & $4.98 \pm 2.72$ & & $1.03 \pm 2.45$ & \\
\hline \multicolumn{5}{|l|}{ Type of ICU } \\
\hline Medical & $4.99 \pm 2.81$ & & $1.12 \pm 2.37$ & \\
\hline Surgery & $4.81 \pm 2.82$ & 0.89 & $0.56 \pm 2.67$ & 0.14 \\
\hline Mixed & $4.90 \pm 2.73$ & & $1.19 \pm 2.45$ & \\
\hline Number of beds in ICU & 0.062 & 0.61 & -0.10 & 0.12 \\
\hline Number of intensivists in ICU & 0.018 & 0.87 & -0.05 & 0.28 \\
\hline \multicolumn{5}{|l|}{ DFLST Protocol available in ICU } \\
\hline Yes & $5.05 \pm 2.80$ & 0.16 & $1.10 \pm 2.37$ & 0.92 \\
\hline No & $4.71 \pm 2.72$ & & $1.11 \pm 2.57$ & \\
\hline \multicolumn{5}{|c|}{ Number of DFLST performed in ICU } \\
\hline$<1 /$ week & $4.70 \pm 2.84$ & 0.12 & $1.15 \pm 2.62$ & 0.75 \\
\hline$\geq 1 /$ week & $5.07 \pm 2.69$ & & $1.08 \pm 2.26$ & \\
\hline
\end{tabular}

DFLST decision to forgo life-sustaining treatment, EOL end-of life, ICU intensive care unit, SD standard deviation 


\begin{tabular}{|c|c|c|c|c|c|c|}
\hline \multicolumn{7}{|l|}{ Themes } \\
\hline & \multicolumn{2}{|c|}{ Wills of end-of-life and relatives } & \multicolumn{2}{|l|}{ Unwanted things } & \multicolumn{2}{|c|}{ Fear of pain and loss of autonomy } \\
\hline \multicolumn{7}{|c|}{ Examples of sentences } \\
\hline & \multicolumn{2}{|c|}{$\begin{array}{l}\text { "I want my wife and children to be } \\
\text { with me in my final moments". / "I } \\
\text { want a priest to be present at my } \\
\text { deathbed". }\end{array}$} & \multicolumn{2}{|c|}{$\begin{array}{l}\text { "I confirm my refusal of all treatment } \\
\text { and therapeutic obstinacy whose sole } \\
\text { aim is to keep me artificially alive". }\end{array}$} & \multicolumn{2}{|c|}{$\begin{array}{l}\text { "I am afraid of unnecessary suffering } \\
\text { if I'm kept alive in a vegetative state". } \\
\text { / "I fear that at the end of my life I } \\
\text { will be a burden to society". }\end{array}$} \\
\hline \multicolumn{7}{|c|}{ Occurrence of word (number) } \\
\hline & I & 48 & Don't want & 8 & To be & 18 \\
\hline & My & 37 & To prolong & 7 & Life & 18 \\
\hline & $\mathrm{Me}$ & 13 & To sustain & 6 & To dread & 6 \\
\hline & Wish & 8 & Treatment & 4 & Condition & 7 \\
\hline & Trust & 5 & Don’t wish & 4 & Suffering & 5 \\
\hline & Wife & 4 & Person & 4 & Alone & 4 \\
\hline & Child & 4 & Year & 3 & Burdensome & 3 \\
\hline & To see & 3 & Goal & 3 & Loss & 3 \\
\hline & To love & 3 & Effect & 3 & Burden & 3 \\
\hline & Health & 3 & Dialysis & 3 & To put & 3 \\
\hline & Priest & 3 & To refuse & 3 & To stay & 3 \\
\hline & Relatives & 3 & Therapeutic obstinacy & 3 & Definitive & 3 \\
\hline & To resuscitate & 3 & Support & 3 & Vegetative & 3 \\
\hline
\end{tabular}

after they had received full and clear information about DFLST from an intensivist. These results are noteworthy because they show the limitations of the use of ADs.

This study highlights the issue of variability between intensivists in the taking of DFLSTs even when ADs are provided. Great variability has been previously reported in several studies [12, 13, 24-27] and attributed to individual physician characteristics such as religion, culture and geographic regions $[7,8]$. The goal of ADs is to enable physicians to comply with the patient's wishes or request, irrespective of their own personal characteristics $[15,16]$. In theory, therefore, ADs should eliminate variability but our findings showed that physicians disagreed about DFLSTs even when ADs were available. There are several possible explanations of this finding. First, the intensivists did not use the ADs to make DFLSTs. However, DFLSTs increased when ADs were available. Second, ADs expressed in free-text boxes led to more possible interpretations of patients' wishes than ADs drawn up in tick-box form, which includes the use of medical terms for instructions that are easily understood by intensivists and provide clear answers to DFLSTs [28]. In studies assessing the interrater reliability for each section of the POLST form using a binary "yes/no" approach, the Kappa coefficients varied from 0.70 to 1.00 [29]. It is possible that the variability among intensivists is low in the binary "yes/no" approach of DFLSTs wishes. However, the possible replies in our study were "yes," "no" and sometimes "partial." Our study differs from these previous reports by the number of questions and the size of the study population, which ensure the robustness of the findings.

A DFLST is a complex but singular process in which the context, the chances of success, the discomfort of treatment, uncertainty regarding prognosis, potential disability and the wishes of the patient are important considerations [30]. ADs with free-text boxes do not allow an exhaustive approach that encompasses in all situations, but give patients the opportunity to express their convictions about the physical or mental impairment [31] that could guide intensivists in their choice of DFLSTs. However, the wording used by the patient can be ambiguous or inappropriate to initiate or to withdraw treatment. The applicability of wishes expressed in ADs results from a match between a hypothetical situation and the medical situation affecting the patient [32]. In our study, the ADs rarely indicated specific wishes about life-sustaining treatments despite information about ICU treatments. This finding could explain the lack of impact of ADs on the high variability between intensivists in the DFLSTs. In this qualitative approach, the influence of relatives or physicians was not assessed. Study of these other areas could improve understanding of the drawing-up, acceptability and usefulness of ADs. The two forms of ADs are complementary and can be associated. After receiving full information during discussion with a healthcare agent, the patient could complete tick-boxes indicating clear DFLSTs that can be implemented whatever the 
situation and use free-text boxes to express values that could help guide the physician in making DFLSTs in situations not previously considered. The decision about resuscitation status is easily made by the patient and can be registered in a tick-box. In contrast, the initiation of renal replacement therapy is an abstract concept that is discussed according to clinical condition and for which the expression of value is more relevant. The surrogate can add nuances and clarifications to the values relayed in ADs so that the best decisions are made according to the specific condition and prognosis of the patient [33]. A surrogate involved in the drafting of values could limit the influence of the intensivist in the decision-making process using ADs [34]. Alternatively, the physician's interpretation of values could be assessed in clinical scenarios. Feedback on DFLSTs made by a sample of intensivists in clinical scenarios would allow the patient to modify ADs so that the intensivists' decisions comply with her or his own and thereby reduce inter-intensivist variability.

Our study has a number of limitations. First, our analyses were performed on the ADs of eight patients. This sample, in agreement with the study feasibility assessment, is not an exhaustive representation of the French population. Nevertheless, the ADs in the study were real documents, which could be used in the decision-making process in clinical practice [35]. Second, to standardize the process, only one intensivist-investigator briefed the patients about DFLSTs and ADs. However, this approach entails the risk of personal influence by the clinician, which can lead to cognitive bias. Third, we do not rule out that the DFLSTs made by intensivists in the scenarios could differ from those taken in everyday practice. However, only a simulation study provides the standardization of situations, which allows assessment of variability between physicians. Our simulation study was time-consuming and unpaid, which could have restricted the participation of some intensivists. The intensivists taking part may have had an interest in ethics and hence were perhaps not representative of the profession as a whole. Fourth, our study design gave no information about family and ICU team discussions or staff opinion. Many DFLSTs are made with non-intensivist physicians, relatives or nurses. Their influence on DFLSTs was not assessed in our study, which focused on the intensivists because they are the main decision-makers for DFLST in the ICU. Fifth, most ADs were collected within 1 month of a single meeting which for some patients could have been too short time to formulate their wishes in full. However, the information about ADs, given by an intensivist-investigator with a video, was of a quality as high as that provided in clinical practice [36] and similar to that used in reference publications $[33,37]$.

\section{Conclusions}

Our study shows that when ADs were available, intensivists were more likely to make DFLSTs. However, ADs did not reduce high inter-intensivist variability in the decision-making process. The great variability observed show that the intensivist's preferences had priority. Further research is needed to establish a process that achieves a better matching of the physicians' decisions with the patient's wishes.

\section{Supplementary Information}

The online version contains supplementary material available at https://doi. org/10.1186/s13054-020-03402-7.

Additional file 1. Characteristics of the 8 patients who wrote advance directives (table).

Additional file 2. The two clinical scenarios (text).

Additional file 3. Flow chart and timing of the study (Figure).

Abbreviations

ADs: Advance directives; DFLST: Decision to forgo life-sustaining treatment; $\mathrm{Cl}$ : Confidence intervals; ICC: Intra-class correlation coefficient; ICU: Intensive care unit; POLST: Physician orders for life-sustaining treatment; RSD: Relative standard deviation.

\section{Acknowledgements}

We are indebted to Mr. Jeffrey Watts for assistance in the preparation of the manuscript. Members of the Facing group: Geoffrey Ledoux (Centre Hospitalier Universitaire de Lille, Médecine Intensive Réanimation Lille); Lea Satrebuisson (Centre Hospitalier Universitaire de Lille, Médecine Intensive Réanimation, Lille); Sophie Six (Centre Hospitalier Universitaire de Lille, Médecine Intensive Réanimation, Lille); Céline Dupre (Centre Hospitalier Universitaire de Lille, Médecine Intensive Réanimation, Lille); Patrick Girardie (Centre Hospitalier Universitaire de Lille, Médecine Intensive Réanimation, Lille); Anahita Rouze (Centre Hospitalier Universitaire de Lille, Médecine Intensive Réanimation, Lille); Erika Decrucq-Parmentier (Centre Hospitalier Universitaire de Lille, Médecine Intensive Réanimation, Lille); Daniel Mathieu (Centre Hospitalier Universitaire de Lille, Médecine Intensive Réanimation, Lille); Julien Poissy (Centre Hospitalier Universitaire de Lille, Médecine Intensive Réanimation, Lille); Sébastien Préau (Centre Hospitalier Universitaire de Lille, Médecine Intensive Réanimation, Lille); Emmanuelle Jaillette (Centre Hospitalier Universitaire de Lille, Médecine Intensive Réanimation, Lille); Raphaël Favory (Centre Hospitalier Universitaire de Lille, Médecine Intensive Réanimation, Lille); Honade Yonis (Centre Hospitalier Universitaire de Lyon, Médecine Intensive Réanimation, Lyon); Romain Hernu (Centre Hospitalier Universitaire de Lyon, Médecine Intensive Réanimation, Lyon); Marie Simon (Centre Hospitalier Universitaire de Lyon, Médecine Intensive Réanimation, Lyon); Thomas Baudry (Centre Hospitalier Universitaire de Lyon, Médecine Intensive Réanimation, Lyon); Thomas Madelaine (Centre Hospitalier Universitaire de Lyon, Médecine Intensive Réanimation, Lyon); Adeline Grateau (Centre Hospitalier Universitaire de Lyon, Médecine Intensive Réanimation, Lyon); Martin Cour (Centre Hospitalier Universitaire de Lyon, Médecine Intensive Réanimation, Lyon); Gérald Chanques (Centre Hospitalier Universitaire de Montpellier, Anesthésie et Réanimation Chirurgicale, Montpellier); Julie Carr (Centre Hospitalier Universitaire de Montpellier, Anesthésie et Réanimation Chirurgicale, Montpellier); Matthieu Conseil (Centre Hospitalier Universitaire de Montpellier, Anesthésie et Réanimation Chirurgicale,

Montpellier); Audrey De Jong (Centre Hospitalier Universitaire de Montpellier, Anesthésie et Réanimation Chirurgicale, Montpellier); Jean-Marc Delay (Centre Hospitalier Universitaire de Montpellier, Anesthésie et Réanimation Chirurgicale, Montpellier); Etienne Imhoff (Centre Hospitalier Universitaire de Montpellier, Anesthésie et Réanimation Chirurgicale, Montpellier); Bruno Souche (Centre Hospitalier Universitaire de Montpellier, Anesthésie et Réanimation Chirurgicale, Montpellier); Marina Thirion (Centre Hospitalier 
Universitaire de Montpellier, Anesthésie et Réanimation Chirurgicale, Montpellier); Aulère Buzançais (Centre Hospitalier Universitaire de Montpellier, Médecine Intensive Réanimation, Montpellier); Boris Jung (Centre Hospitalier Universitaire de Montpellier, Médecine Intensive Réanimation, Montpellier); Vincent Bruno (Centre Hospitalier Universitaire de Montpellier, Médecine Intensive Réanimation, Montpellier); Delphine Daubin (Centre Hospitalier Universitaire de Montpellier, Médecine Intensive Réanimation, Montpellier); Laura Platon (Centre Hospitalier Universitaire de Montpellier, Médecine Intensive Réanimation, Montpellier); Jean-Jacques Tudesq (Centre Hospitalier Universitaire de Montpellier, Médecine Intensive Réanimation, Montpellier); Liliane Landreau (Centre Hospitalier Universitaire de Montpellier, Médecine Intensive Réanimation, Montpellier); Henri Boby (Centre Hospitalier Universitaire de Clermont-Ferrand, Anesthésie et Réanimation Chirurgicale, Clermont-Ferrand); Nicolas Rascol (Centre Hospitalier Universitaire de Clermont-Ferrand, Anesthésie et Réanimation Chirurgicale, Clermont-Ferrand); Lucia Uta (Centre Hospitalier Universitaire de Clermont-Ferrand, Anesthésie et Réanimation Chirurgicale, Clermont-Ferrand); Vedat Eljezi (Centre Hospitalier Universitaire de Clermont-Ferrand, Anesthésie et Réanimation Chirurgicale, Clermont-Ferrand); Mélanie Chasteloux (Centre Hospitalier Universitaire de Clermont-Ferrand, Anesthésie et Réanimation Chirurgicale, Clermont-Ferrand); Nicolas Coullier (Centre Hospitalier Universitaire de Clermont-Ferrand, Anesthésie et Réanimation Chirurgicale, Clermont-Ferrand); Laurent Nicolet (Centre Hospitalier Universitaire de Nantes, Médecine Intensive Réanimation, Nantes); Charlotte Garret (Centre Hospitalier Universitaire de Nantes, Médecine Intensive Réanimation, Nantes); Noëlle Brule (Centre Hospitalier Universitaire de Nantes, Médecine Intensive Réanimation, Nantes); Agathe Delbove (Centre Hospitalier Universitaire de Nantes, Médecine Intensive Réanimation, Nantes); Maelle Martin (Centre Hospitalier Universitaire de Nantes, Médecine Intensive Réanimation, Nantes); Jean-Batptiste Lascarrou (Centre Hospitalier Universitaire de Nantes, Médecine Intensive Réanimation, Nantes); François Bougerol (Centre Hospitalier de Dieppe, Réanimation, Dieppe); Pierre-Louis Declerca (Centre Hospitalier de Dieppe, Réanimation, Dieppe); Marion Beuzelin (Centre Hospitalier de Dieppe, Réanimation, Dieppe); Jean-Pierre Eraldi (Centre Hospitalier de Dieppe, Réanimation, Dieppe); Antoine Marchalot (Centre Hospitalier de Dieppe, Réanimation, Dieppe); Thomas Poussant (Centre Hospitalier de Chalon-sur-Saône, Réanimation, Chalon-sur-Saône); Maël Hamet (Centre Hospitalier de Chalon-sur-Saône, Réanimation, Chalon-surSaône); Martial Delorme (Centre Hospitalier de Chalon-sur-Saône, Réanimation, Chalon-sur-Saône); Adrien Lhoumeau (Centre Hospitalier de Chalon-surSaône, Réanimation, Chalon-sur-Saône); Jen Darphin (Centre Hospitalier de Chalon-sur-Saône, Réanimation, Chalon-sur-Saône); Julien Pascal (Centre Hospitalier Universitaire de Clermont-Ferrand, Anesthésie et Réanimation Chirurgicale, Clermont-Ferrand); Charlotte Fernandez-Canal (Centre Hospitalier Universitaire de Clermont-Ferrand, Anesthésie et Réanimation Chirurgicale, Clermont-Ferrand); Aurélien Momon (Centre Hospitalier Universitaire de Clermont-Ferrand, Anesthésie et Réanimation Chirurgicale, Clermont-Ferrand); Julien Marin (Centre Hospitalier Universitaire de Clermont-Ferrand, Anesthésie et Réanimation Chirurgicale, Clermont-Ferrand); Laure Calvet (Centre Hospitalier Universitaire de Clermont-Ferrand, Médecine Intensive Réanimation, Clermont-Ferrand); Edouard Soum (Centre Hospitalier Universitaire de Clermont-Ferrand, Médecine Intensive Réanimation, Clermont-Ferrand); Claire Bachelier (Centre Hospitalier Universitaire de Clermont-Ferrand, Médecine Intensive Réanimation, Clermont-Ferrand); Kostas Bachoumas (Centre Hospitalier Universitaire de Clermont-Ferrand, Médecine Intensive Réanimation, Clermont-Ferrand); Thomas Godet (Centre Hospitalier Universitaire de Clermont-Ferrand, Anesthésie et Réanimation Chirurgicale, Clermont-Ferrand); Danièle Saurel (Centre Hospitalier Universitaire de Clermont-Ferrand, Anesthésie et Réanimation Chirurgicale, Clermont-Ferrand); Marie Vignaud (Centre Hospitalier Universitaire de Clermont-Ferrand, Anesthésie et Réanimation Chirurgicale, Clermont-Ferrand); Marc Gainnier (Centre Hospitalier Universitaire de Marseille, Médecine Intensive Réanimation, Marseille); Dominique Lambert (Centre Hospitalier Universitaire de Marseille, Médecine Intensive Réanimation, Marseille); Lucas Benarous (Centre Hospitalier Universitaire de Marseille, Médecine Intensive Réanimation, Marseille); Julien Carvelli (Centre Hospitalier Universitaire de Marseille, Médecine Intensive Réanimation, Marseille); Philippe Letocart (Centre Hospitalier de Rodez, Médecine Intensive Réanimation, Rodez); Arnaud Delahaye (Centre Hospitalier de Rodez, Médecine Intensive Réanimation Rodez); Sophie Dufraisse (Centre Hospitalier de Rodez, Médecine Intensive Réanimation, Rodez); François Lenfant (Centre Hosptialier de Cannes, Médecine Intensive Réanimation, Cannes); Raphaël Chambon (Centre
Hospitalier de Cannes, Médecine Intensive Réanimation, Cannes); Jerôme Bedel (Centre Hospitalier de Cannes, Médecine Intensive Réanimation, Cannes); Nicolas François (Centre Hospitalier de Montluçon, Réanimation, Montluçon); Thierry Comte (Centre Hospitalier de Montluçon, Réanimation, Montluçon); Pierre Couhault (Centre Hospitalier de Montluçon, Réanimation, Montluçon); Jean-Baptiste Roudaut (Centre Hospitalier Universitaire de Dijon, Médecine Intensive Réanimation, Dijon); Auguste Dargent (Centre Hospitalier Universitaire de Dijon, Médecine Intensive Réanimation, Dijon); Gwenaël Prat (Centre Hospitalier Universitaire de Brest, Médecine Intensive Réanimation, Brest); Vanessa Jean-Michel (Centre Hospitalier Universitaire de Brest, Médecine Intensive Réanimation, Brest); Beatrice Fourgeaud (Centre de Lutte Contre le Cancer Jean Perrin, Anesthésie et Réanimation Chirurgicale, Clermont-Ferrand); Charlotte Baud (Centre de Lutte Contre le Cancer Jean Perrin, Anesthésie et Réanimation Chirurgicale, Clermont-Ferrand); Cédric Darreau (Centre Hospitalier Universitaire de Angers, Médecine Intensive Réanimation, Angers); Pierre-Yves Olivier (Centre Hospitalier Universitaire de Angers, Médecine Intensive Réanimation, Angers); Mourad Benyamina (Centre Hosptitalier Universitaire Saint-Louis, Centre de traitement des Brûlés, Paris); François Depret (Centre Hosptitalier Universitaire Saint-Louis, Centre de traitement des Brûlés, Paris); Hervé Hyvernat (Centre Hospitalier Universitaire de Nice, Médecine Intensive Réanimation, Nice); Pierre-Eric Danin (Centre Hospitalier Universitaire de Nice, Médecine Intensive Réanimation, Nice); Nordine Ait Ben Said (Centre Hospitalier Universitaire de Clermont-Ferrand, Anesthésie et Réanimation Chirurgicale, Clermont-Ferrand); Raiko Blondonnet (Centre Hospitalier Universitaire de Clermont-Ferrand, Anesthésie et Réanimation Chirurgicale, Clermont-Ferrand); Clémence Deville (Centre Hospitalier Universitaire de Clermont-Ferrand, Nephrology, Clermont-Ferrand); Aurélien Mulliez (Centre Hospitalier Universitaire de Clermont-Ferrand, Service de Biostastistiques, Clermont-Ferrand); Pierre Cornillon (Clermont-Ferrand); David Brugnon (Centre Hospitalier Universitaire de Clermont-Ferrand, Soins palliatifs, Clermont-Ferrand); Lise Vernis (Centre Hospitalier Universitaire de Clermont-Ferrand, Anesthésie et Réanimation Chirurgicale, Clermont-Ferrand); Delphine Aguettaz (Centre Hospitalier Universitaire de Clermont-Ferrand, Cardiologie, Clermont-Ferrand); Lise Thibonnier (Centre Hospitalier Universitaire de Clermont-Ferrand, Pneumologie, Clermont-Ferrand); Hugues François (Centre Hospitalier Universitaire de Clermont-Ferrand, Pneumologie, Clermont-Ferrand); Frédérique Martins-Condé (Centre Hospitalier Universitaire de Clermont-Ferrand, Gériatrie, Clermont-Ferrand); Maeva Wongfat (Pole santé république, Néphrologie, Clermont-Ferrand); Ibrahim Moustapha (Centre Hospitalier Universitaire de Grenoble, Médecine Intensive Réanimation, Grenoble); Thibaud Pranal (Centre Hospitalier de Vichy, Réanimation, Vichy); François Brenat (Centre Hospitalier du Puy-en-Velay, Réanimation, Le Puy-en-Velay); Bertrand Combres (Centre Hospitalier d'Albi, Réanimation, Albi); Anne Veinstein (Centre Hospitalier Universitaire de Poitiers, Médecine Intensive Réanimation, Poitiers); Alexandre Boyer (Centre Hospitalier Universitaire de Bordeaux, Médecine Intensive Réanimation, Bordeaux).

\section{Authors' contributions}

MS, AL and BP conceived and designed the study; MS, MJ, JCR, LA, SJ, KK, $A M$, JR, JPR, JMD, RC, BS, JB, JD, PMB, PV, JPQ, CA, NE, PA, AF, JD, NT, JMC and $R G$ recruited the participants and collected the data; $M S, A V L, V G, C L, R G, A L$ and $\mathrm{BP}$ analyzed and interpreted data; MS, AL and BP drafted the report and all authors contributed to review it. All authors approved the final version. All authors read and approved the final manuscript.

\section{Funding}

No Grant.

\section{Availability of data and materials}

The data that support the findings of this study are available from the corresponding author upon reasonable request.

\section{Ethical approval and consent to participate}

Approved by the Institutional Review Board of the Comité Pour la Protection des Personnes Sud-Est 6 (IRB00008526; No.2016/CE87).

\section{Consent for publication}

Not applicable.

\section{Competing interests}

The others authors have no financial conflict of interest related to this study. 


\section{Author details}

${ }^{1}$ Department of Anaesthesiology and Critical Care Medicine, Estaing Hospital, University Hospital of Clermont-Ferrand, Clermont-Ferrand, France. ${ }^{2}$ INSERM U1190, CHU Lille, Department of Critical Care Medicine, Roger Salengro Hospital, Univ. Lille, 59000 Lille, France. ${ }^{3}$ Palliative Care Unit, Louise Michel Hospital, University Hospital of Clermont-Ferrand, Clermont-Ferrand, France. ${ }^{4}$ Biostatistics Unit (DRCI), University Hospital of Clermont-Ferrand, Clermont-Ferrand, France. ${ }^{5}$ Medical Intensive Care Unit, La Croix Rousse Hospital, University Hospital of Lyon, Lyon, France. ${ }^{6}$ Medical Intensive Care Unit, Edouard Herriot Hospital, University Hospital of Lyon, Lyon, France. ${ }^{7}$ Department of Anaesthesiology and Critical Care Medicine, Saint Eloi Hospital, University Hospital of Montpellier, Montpellier, France. ${ }^{8}$ Medical Intensive Care Unit, Lapeyronnie Hospital, University Hospital of Montpellier, Montpellier, France. ${ }^{9}$ Cardiac Surgery Intensive Care Unit, Department of Anaesthesiology and Critical Care Medicine, Montpied Hospital, University Hospital of Clermont-Ferrand, Clermont-Ferrand, France. ${ }^{10}$ Medical Intensive Care Unit, Hotel-Dieu Hospital, University Hospital of Nantes, Nantes, France. ${ }^{11}$ Intensive Care Unit, Pasteur Hospital, Hospital of Dieppe, Dieppe, France. ${ }^{12}$ Intensive Care Unit, Morey Hospital, Hospital of Chalon-Sur-Saône, Chalon-sur-Saône, France. ${ }^{13}$ Neurocritical Care Unit, Department of Anaesthesiology and Critical Care Medicine, Montpied Hospital, University Hospital of Clermont-Ferrand, Clermont-Ferrand, France. ${ }^{14}$ Medical Intensive Care Unit, Montpied Hospital, University Hospital of Clermont-Ferrand, Clermont-Ferrand, France. ${ }^{15}$ Emergency Intensive Care Unit, La Timone Hospital, University Hospital of Marseille, Marseille, France. ${ }^{16}$ Intensive Care Unit, Puel Hospital, Hospital of Rodez, Rodez, France. ${ }^{17}$ Intensive Care Unit, Veil Hospital, Hospital of Cannes, Cannes, France. ${ }^{18}$ Intensive Care Unit, Hospital of Montluçon, Montluçon, France. ${ }^{19}$ Medical Intensive Care Unit, Mitterrand Hospital, University Hospital of Dijon, Dijon, France. ${ }^{20}$ Medical Intensive Care Unit, Centre Hospitalier Universitaire de Brest, Université de Bretagne Occidentale, Brest, France. ${ }^{21}$ Intensive Care Unit, Centre Jean Perrin, 54 Rue Montalembert, BP69, 63003 Clermont-Ferrand, Cedex 1, France. ${ }^{22}$ Medical Intensive Care Unit, Larrey Hospital, University Hospital of Angers, Angers, France. ${ }^{23}$ Intensive Care Unit, Department of Anaesthesiology and Critical Care Medicine, Saint-Louis Hospital, Assistance Publique Hopitaux de Paris, Paris, France. ${ }^{24}$ Medical Intensive Care Unit,l'Archet Hospital, University Hospital of Nice, Nice, France. ${ }^{25}$ Medical Intensive Care Unit, Michallon Hospital, University Hospital of Grenoble, Grenoble, France. ${ }^{26}$ GRC 29, AP-HP, DMU DREAM, Department of Anaesthesiology and Critical Care, Pitié-Salpêtrière Hospital, Sorbonne University, Paris, France. ${ }^{27}$ UPU ACCePPt, Université Clermont Auvergne, Clermont-Ferrand, France. ${ }^{28}$ EA-481, Laboratoire de Neurosciences, UBFC, Besançon, France. ${ }^{29}$ Intensive Care Unit, Department of Anaesthesiology and Critical Care Medicine, Estaing Hospital, University Hospital of Clermont-Ferrand, Clermont-Ferrand, France. ${ }^{30}$ LMGE «Laboratoire Micro-Organismes: Génome Et Environnement», UMR CNRS 6023, Clermont-Auvergne University, Clermont-Ferrand, France. ${ }^{31}$ Intensive Care Medicine, Montpied Teaching Hospital, 54 Rue Montalembert, BP69, 63003 Clermont-Ferrand, Cedex 1, France.

Received: 27 August 2020 Accepted: 20 November 2020 Published online: 02 December 2020

\section{References}

1. Lobo SM, De Simoni FHB, Jakob SM, Estella A, Vadi S, Bluethgen A, et al. Decision-making on withholding or withdrawing life support in the ICU: a worldwide perspective. Chest. 2017;152:321-9.

2. Lautrette A, Garrouste-Orgeas M, Bertrand P-M, Goldgran-Toledano D, Jamali S, Laurent $V$, et al. Respective impact of no escalation of treatment, withholding and withdrawal of life-sustaining treatment on ICU patients' prognosis: a multicenter study of the Outcomerea Research Group. Intensive Care Med. 2015;41:1763-72.

3. Ciroldi M, Cariou A, Adrie C, Annane D, Castelain V, Cohen Y, et al. Ability of family members to predict patient's consent to critical care research. Intensive Care Med. 2007:33:807-13.

4. Lautrette A, Peigne V, Watts J, Souweine B, Azoulay E. Surrogate decision makers for incompetent ICU patients: a European perspective. Curr Opin Crit Care. 2008;14:714-9.

5. White DB, Ernecoff N, Buddadhumaruk P, Hong S, Weissfeld L, Curtis JR, et al. Prevalence of and factors related to discordance about prognosis between physicians and surrogate decision makers of critically ill patients. JAMA. 2016;315:2086-94.

6. Luce JM. A history of resolving conflicts over end-of-life care in intensive care units in the United States. Crit Care Med. 2010;38:1623-9.

7. Sprung $\mathrm{CL}$, Cohen SL, Sjokvist P, Baras M, Bulow H-H, Hovilehto S, et al. End-of-life practices in European intensive care units: the Ethicus Study. JAMA. 2003;290:790-7.

8. Sprung CL, Woodcock T, Sjokvist P, Ricou B, Bulow H-H, Lippert A, et al. Reasons, considerations, difficulties and documentation of end-of-life decisions in European intensive care units: the ETHICUS Study. Intensive Care Med. 2008;34:271-7.

9. Valley TS, Admon AJ, Zahuranec DB, Garland A, Fagerlin A, Iwashyna TJ. Estimating ICU benefit: a randomized study of physicians. Crit Care Med. 2019;47:62-8.

10. Barnato AE, Hsu HE, Bryce CL, Lave JR, Emlet LL, Angus DC, et al. Using simulation to isolate physician variation in intensive care unit admission decision making for critically ill elders with end-stage cancer: a pilot feasibility study. Crit Care Med. 2008;36:3156-63.

11. Kruser JM, Aaby DA, Stevenson DG, Pun BT, Balas MC, Barnes-Daly MA, et al. Assessment of variability in end-of-life care delivery in intensive care units in the United States. JAMA Netw Open. 2019;2:e1917344.

12. Hart JL, Harhay MO, Gabler NB, Ratcliffe SJ, Quill CM, Halpern SD. Variability among US intensive care units in managing the care of patients admitted with preexisting limits on life-sustaining therapies. JAMA Intern Med. 2015;175:1019-26.

13. Curtis JR, Engelberg RA, Teno JM. Understanding variability of end-of-life care in the ICU for the elderly. Intensive Care Med. 2017:43:94-6.

14. Wunsch H, Harrison DA, Harvey S, Rowan K. End-of-life decisions: a cohort study of the withdrawal of all active treatment in intensive care units in the United Kingdom. Intensive Care Med. 2005;31:823-31.

15. Silveira MJ, Kim SYH, Langa KM. Advance directives and outcomes of surrogate decision making before death. N Engl J Med. 2010;362:1211-8.

16. Schenker Y, White DB, Arnold RM. What should be the goal of advance care planning? JAMA Intern Med. 2014;174:1093-4.

17. Sprung CL, Truog RD, Curtis JR, Joynt GM, Baras M, Michalsen A, et al. Seeking worldwide professional consensus on the principles of endof-life care for the critically ill. The consensus for worldwide end-of-life practice for patients in intensive care units (WELPICUS) study. Am J Respir Crit Care Med. 2014;190:855-66.

18. von Elm E, Altman DG, Egger M, Pocock SJ, Gøtzsche PC, Vandenbroucke $J$, et al. The strengthening the reporting of observational studies in epidemiology (STROBE) statement: guidelines for reporting observational studies. Lancet Lond Engl. 2007;370:1453-7.

19. Jackson GG, Arana-Sialer JA, Andersen BR, Grieble HG, McCABE WR. Profiles of pyelonephritis. Arch Intern Med. 1962;110:63-75.

20. Appelbaum PS. Clinical practice. Assessment of patients' competence to consent to treatment. N Engl J Med. 2007;357:1834-40.

21. https://www.has-sante.fr/upload/docs/application/pdf/2016-11/da_ formulaire_v2_maj.pdf

22. (2016) Ministère des Affaires Sociales et de la Santé: Arrêté du 3 août 2016 relatif au modèle de directives anticipées prévu à l'article L. 1111-11 du code de la santé publique. J Officiel 2016;0181

23. Katz S. Assessing self-maintenance: activities of daily living, mobility, and instrumental activities of daily living. J Am Geriatr Soc. 1983;31:721-7.

24. Mark NM, Rayner SG, Lee NJ, Curtis JR. Global variability in withholding and withdrawal of life-sustaining treatment in the intensive care unit: a systematic review. Intensive Care Med. 2015;41:1572-85.

25. Alemayehu E, Molloy DW, Guyatt GH, Singer J, Penington G, Basile J, et al. Variability in physicians' decisions on caring for chronically ill elderly patients: an international study. CMAJ Can Med Assoc J J Assoc Medicale Can. 1991;144:1133-8.

26. Wunsch $H$, Angus DC, Harrison DA, Collange O, Fowler R, Hoste EAJ, et al. Variation in critical care services across North America and Western Europe. Crit Care Med. 2008;36(2787-93):e1-9.

27. Quill CM, Ratcliffe SJ, Harhay MO, Halpern SD. Variation in decisions to forgo life-sustaining therapies in US ICUs. Chest. 2014;146:573-82.

28. Halpern SD, Small DS, Troxel AB, Cooney E, Bayes B, Chowdhury M, et al. Effect of default options in advance directives on hospital-free days and care choices among seriously ill patients: a randomized clinical trial. JAMA Netw Open. 2020;3:e201742. 
29. Lovadini GB, Fukushima FB, Schoueri JFL, Dos Reis R, Fonseca CGF, Rodriguez JJC, et al. Evaluation of the interrater reliability of end-of-life medical orders in the physician orders for life-sustaining treatment form. JAMA Netw Open. 2019;2:e192036.

30. Guidet B, Hodgson E, Feldman C, Paruk F, Lipman J, Koh Y, et al. The Durban World Congress Ethics Round Table Conference Report: II. Withholding or withdrawing of treatment in elderly patients admitted to the intensive care unit. J Crit Care. 2014;29:896-901.

31. Gaster B, Larson EB, Curtis JR. Advance directives for dementia: meeting a unique challenge. JAMA. 2017;318:2175-6.

32. Hartog CS, Peschel I, Schwarzkopf D, Curtis JR, Westermann I, Kabisch B, et al. Are written advance directives helpful to guide end-of-life therapy in the intensive care unit? A retrospective matched-cohort study. J Crit Care. 2014;29:128-33.

33. Garrouste-Orgeas M, Tabah A, Vesin A, Philippart F, Kpodji A, Bruel C, et al. The ETHICA study (part II): simulation study of determinants and variability of ICU physician decisions in patients aged 80 or over. Intensive Care Med. 2013;39:1574-83.

34. Chiarchiaro J, Ernecoff NC, Scheunemann LP, Hough CL, Carson SS, Peterson MW, et al. Physicians rarely elicit critically ill patients' previously expressed treatment preferences in intensive care units. Am J Respir Crit Care Med. 2017;196:242-5.

35. Council of Europe. Recommendation CM/Rec(2009) 11 of the Committee of Ministers to member states on principles continuing powers of attorney and advance directives for incapacity. Eur J Health Law. 2010;17:205-10.

36. Miki R, Becker CB, Ide K, Kawakami K. Timing and facilitation of advanced directives in Japan. Arch Gerontol Geriatr. 2018;79:83-7.

37. Philippart F, Vesin A, Bruel C, Kpodji A, Durand-Gasselin B, Garçon P, et al. The ETHICA study (part I): elderly's thoughts about intensive care unit admission for life-sustaining treatments. Intensive Care Med. 2013;39:1565-73.

\section{Publisher's Note}

Springer Nature remains neutral with regard to jurisdictional claims in published maps and institutional affiliations.
Ready to submit your research? Choose BMC and benefit from:

- fast, convenient online submission

- thorough peer review by experienced researchers in your field

- rapid publication on acceptance

- support for research data, including large and complex data types

- gold Open Access which fosters wider collaboration and increased citations

- maximum visibility for your research: over $100 \mathrm{M}$ website views per year

At BMC, research is always in progress.

Learn more biomedcentral.com/submissions 